\title{
Proposal and Analysis of Wavelength-Switchable Optical Fiber Filter Based on a Solc Type
}

\author{
Minwook Kim and Jaehoon Jung* \\ Department of Electronics and Electrical Engineering, Dankook University, Yongin-Si, 448-701, Korea
}

(Received June 18, 2008 : revised July 15, 2008 : accepted July 16, 2008)

\begin{abstract}
This paper proposes a new polarization-independent Sagnac birefringence loop structure-based multiwavelength-periodic filter and presents measurements and analysis of its spectrum. The filter can be used in several schemes by adjusting the orientation angles of two quarter waveplates and the operating characteristics in the reflection type are analyzed including dispersion and polarization mode dispersion at each principal axis. This filter has polarization-independent spectra but a polarization-dependent dispersion, consequently polarization mode dispersion whose values changes with operating schemes.
\end{abstract}

Keywords: mulitwavelength-periodic filter, dispersion, polarization mode dispersion

OCIS codes : (060.0060) Fiber optics and optical communications ; (060.2330) Fiber optics communications ; (060.2340) Fiber optics components

\section{INTRODUCTION}

Recently, fiber birefringent filters based on a Sagnac birefringence loop structure (SBLS) have received a great deal of attention and have been proven to be an effective tool for a switching device. The theory and applications of Sagnac fiber loop reflectors, fabricated by forming a fiber loop between the output ports of a directional coupler, were theoretically described [1] and are wellunderstood. And it has been finding applications in many different areas of photonics. Meanwhile, it was realized that by inserting a high birefringent fiber (HBF) in the Sagnac fiber loop, that is, by forming an SBLS, multiwavelength-periodic filtering function could be produced. Techniques for building optical filters using an SBLS (Sagnac birefringence filter) have been proposed and experimentally demonstrated in various forms $[2,3]$.

In this paper, a new polarization-independent SBLSbased multiwavelength-periodic filter is proposed and described, which can make its channel wavelength switchable or tunable by adjusting polarization controllers ( $\mathrm{P}$ C's) within it without doing any physical harm to the durability of the HBF [4]. The characteristics including reflection spectrum and dispersion for the proposed SB LS-based multiwavelength-switchable filter are analyzed.

*Corresponding author: andyjung@dku.edu
The spectral response of the proposed filter was computed by using the Jones-matrix method.

\section{ANALYSIS OF REFLECTION SPECTRA FOR MULTIWAVELENGTH-SWITCHABLE FILTER}

A new polarization-independent SBLS-based multi wavelength-periodic filter was proposed and operating conditions were described ' which can make its channel wavelength switchable (specifically, interleaving) by adjusting quarter waveplates (QWP's) within the filter [4]. The filter consists of a polarization beam splitter (PBS: OZ Optics) and a Sagnac birefringence loop that is composed of HBF's, 50:50 coupler, and two QWP's (OZ Optics). The schematic diagram of the filter is shown in Fig. 1. The PBS is a compact optical apparatus which can split the light into two orthogonal polarization components or combine them into one output fiber. And the Sagnac birefringence loop in the dotted box in Fig. 1 acts as a polarization-independent multiwavelengthperiodic filter [2].

If we assume ideal 50:50 coupler, QWP's, and PBS and any insertion loss due to optical components which comprise the filter is not considered, corresponding reflectivity $\mathrm{R}$ and transmittivity $\mathrm{T}$ of the filter can be 


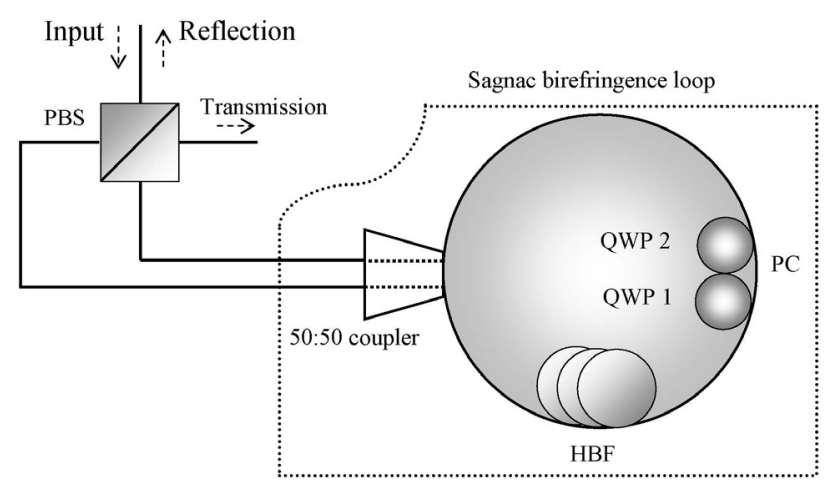

FIG. 1. Schematic diagram of the SBLS-based multiwave length- switchable filter (dots: splicing points between the $\mathrm{HBF}$ and SMF

calculated as follows.

$$
\begin{aligned}
R= & 1-\left[\sin \left(\theta_{1}-\theta_{2}\right) \cos \left(\theta_{1}-\theta_{2}\right) \cos \left(\frac{\Gamma}{2}\right)\right. \\
& \left.+\sin \left(2 \theta_{p}-\theta_{1}-\theta_{2}\right) \cos \left(\theta_{1}-\theta_{2}\right) \sin \left(\frac{\Gamma}{2}\right)\right]^{2} \\
T= & {\left[\sin \left(\theta_{1}-\theta_{2}\right) \cos \left(\theta_{1}-\theta_{2}\right) \cos \left(\frac{\Gamma}{2}\right)\right.} \\
& \left.+\sin \left(2 \theta_{p}-\theta_{1}-\theta_{2}\right) \cos \left(\theta_{1}-\theta_{2}\right) \sin \left(\frac{\Gamma}{2}\right)\right]^{2}
\end{aligned}
$$

where $\theta_{1}$ and $\theta_{2}$ are, respectively, fast-axis orientation (azimuthal) angles of the QWP 1 and 2 with respect to the horizontal axis of the PBS (which is assumed as the reference axis) and $\theta_{\mathrm{p}}$ is the orientation angle of the fast axis of the HBF with respect to the reference axis. And $\Gamma(=2 \pi \mathrm{BL} / \lambda)$ is the phase difference between the fast and slow axis components of the light at the free space wavelength $\lambda$, which propagates through the HBF with the birefringence $B$ and length $L$. Depending on the set of $\theta_{1}$ and $\theta_{2}$, both amplitude (modulation

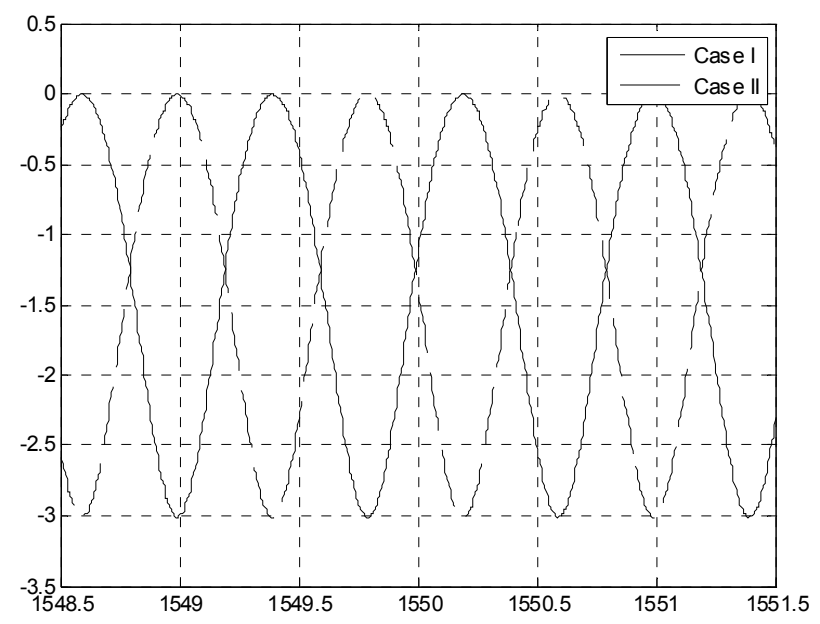

FIG. 2. (a) Calculated reflection spectra of the SBLSbased multiwavelength-switchable filter at optimal settings. depth) and maxima/minima transmission wavelengths of interference pattern in filter spectrum change. From these two equations, we know that the filter has reflection and transmission spectrum independent of input polarization.

The operating schemes of this filter can be categorized as several types. The optimal setting is defined as a reflection-type interleaving filter. For the interleaving filter, two interleaved interference spectra like $\pi$ phaseshifted spectrum pair can be achieved at suitably chosen two sets of $\theta_{1}$ and $\theta_{2}$, designated as optimal sets.

Figure 2 (a) shows the calculated reflection spectra of the filter at two optimal sets of $\left(\theta_{1}, \theta_{2}\right)$ for interleaving operation:

solid line (Case I)

$$
\begin{aligned}
& \left(\theta_{p}-\left\{(n-m+1)-\frac{1}{4}\left[(-1)^{n}-(-1)^{m}\right]\right\} \frac{\pi}{2},\right. \\
& \left.\theta_{p}-\left\{(n+m-1)-\frac{1}{4}\left[(-1)^{n}+(-1)^{m}\right]\right\} \frac{\pi}{2}\right)
\end{aligned}
$$

and dashed line (Case II)

$$
\begin{aligned}
& \left(\theta_{p}-\left\{(n-m)-\frac{1}{4}\left[(-1)^{n}-(-1)^{m}\right]\right\} \frac{\pi}{2},\right. \\
& \left.\theta_{p}-\left\{(n+m)-\frac{1}{4}\left[(-1)^{n}+(-1)^{m}\right]\right\} \frac{\pi}{2}\right)
\end{aligned}
$$

where $n$ and $m$ are integers. Figure 2 (b) shows the measured reflection spectra of the filter at two optimal sets. The measured data correspond well with those calculated except for some loss. In the experiment, we used Bow-tie type HBF whose birefringence and length are $\sim 4.8 \times 10^{-4}$ and $6.25 \mathrm{~m}$, respectively. The length of the HBF was determined so that wavelength spacing (channel spacing) between fringe maxima becomes 0.8 $\mathrm{nm}$. Slight differences from the theoretical result in am-

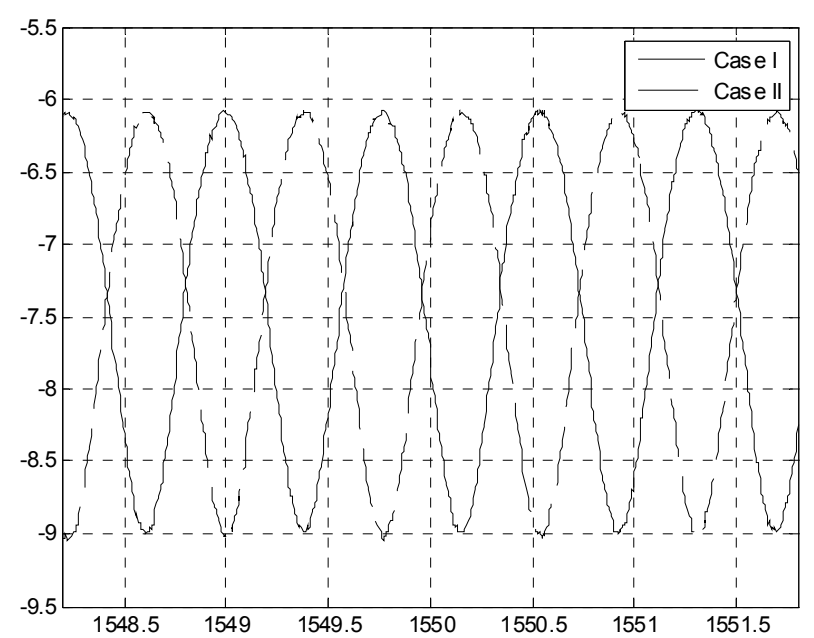

FIG. 2. (b) Measured reflection spectra of the SBLSbased multiwavelength-switchable filter at optimal settings. 


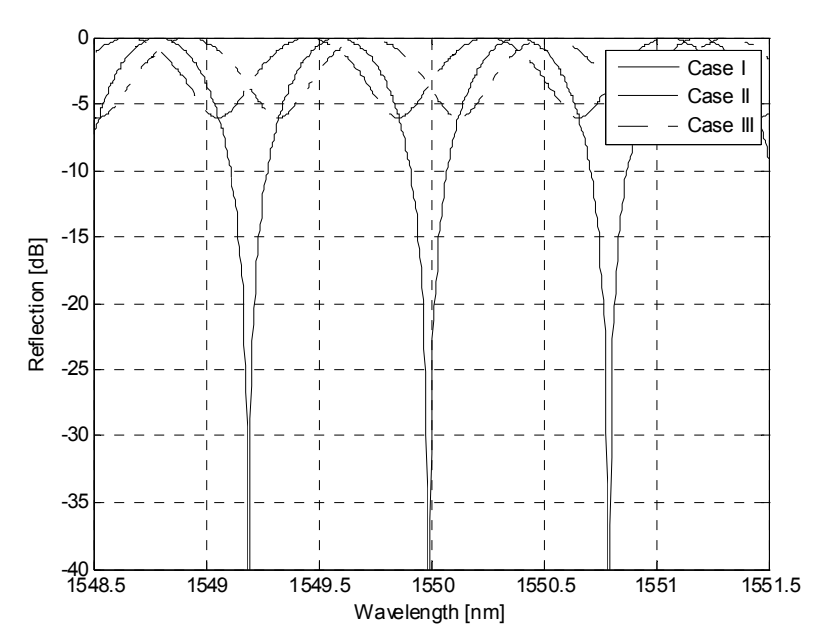

FIG. 3. Calculated reflection spectra of the filter at some other non-optimal settings.

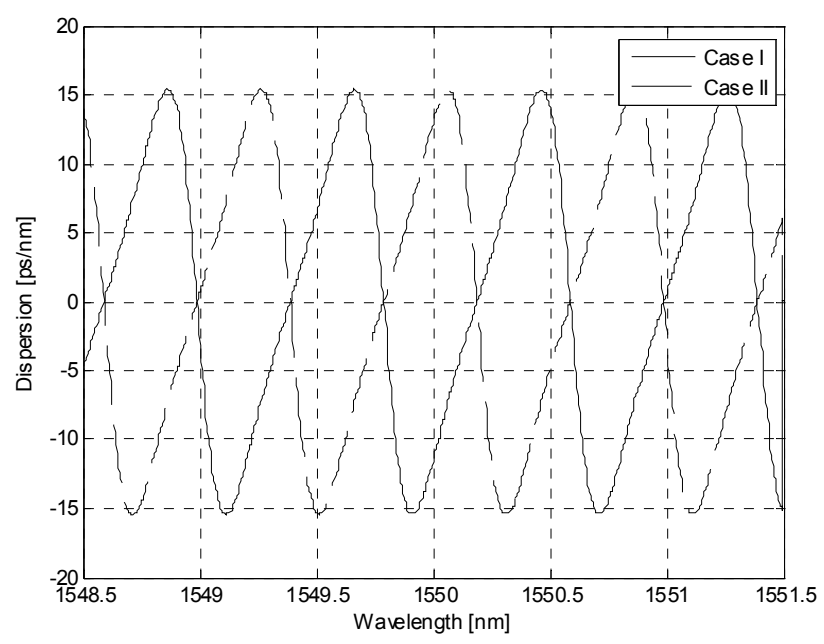

(a) fast axis

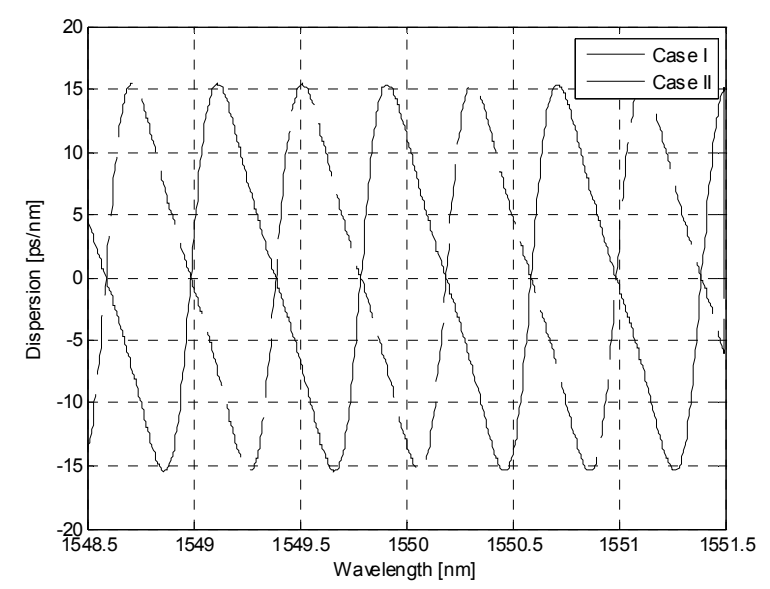

(b) slow axis

FIG. 4. Calculated reflection dispersion of the SBLSbased multiwavelength-switchable filter at optimal settings. plitude and wavelength location stem from the slight unevenness in insertion loss of the 50:50 coupler and PBS and the value of $\theta_{p}$ different from that used in the calculation, respectively. The typical channel isolation was measured to be $\sim 3 \mathrm{~dB}$. Insertion loss was measured to be less than $\sim 6.1 \mathrm{~dB}$, which mainly comes from that of two QWP's (totally $\sim 1.5 \mathrm{~dB})$, PBS $(\sim 0.93 \mathrm{~dB}$ for one way, totally $\sim 1.86 \mathrm{~dB})$, coupler loss $(\sim 0.58 \mathrm{~dB}$ for one way, totally $\sim 1.16 \mathrm{~dB})$, and fiber fusion splicing loss $(\sim 1.47 \mathrm{~dB})$ between the HBF and SMF. The insertion loss can be reduced by using low-loss waveplates and PBS and by improving the fusion splicing of fiber splices between the HBF and SMF. Especially, in order to examine the input polarization independence of the filter, the polarization sensitivity of the output spectrum was measured by placing an additional PC (Agilent 8169 A) in front of the lead-in fiber (input port of the filter).

For general combinations except optimal sets, the filter has spectral characteristics like those shown in Fig. 3. The solid line is obtained at the combinations of QWP angles $\left(\theta_{1}, \theta_{2}\right)$ such as $\left(\theta_{p}-\left(\frac{2 n-1}{4}\right) \pi, \theta_{p}-\left(\frac{2 n-1}{4}\right) \pi\right)$ (Case $\mathrm{I})$, where $\mathrm{n}$ is integer. While the channel isolation of the filter is large (more than $30 \mathrm{~dB}$ ) in these combinations, there is no combination of QWP angles which makes the reflection spectrum have the same channel isolation and interleaved channel location, compared with those of the spectrum plotted in a solid line. The dashed and dashed-dotted lines are obtained at the combinations of $\left(\theta_{p}-\frac{\pi}{4}, \theta_{p}-\frac{\pi}{12}\right)\left(\right.$ Case $\Pi$ I) and $\left(\theta_{p}-\frac{\pi}{4}, \theta_{p}-\frac{5 \pi}{12}\right)$ (Case III), respectively. Same channel isolation at two different channel locations can be achieved at a pair of QWP combinations.

\section{DISPERSION AND POLARIZATION MODE DISPERSION CHARACTERISTICE}

Let $U(z, t)$ is the slowly varying amplitude of the pulse envelop and dispersions of optical devices are calculated as follows;

$$
\begin{aligned}
& U(z, t)=A(\omega) e^{j(\omega t-k z)}=|A(\omega)| e^{j(\omega t-k z+\phi)} \\
& D=\frac{d t_{g}}{d \lambda}=\frac{d}{d \lambda}\left(\frac{d \phi}{d \omega}\right)=\frac{d}{d \lambda}\left(-\frac{\lambda^{2}}{2 \pi c} \frac{d \phi}{d \lambda}\right)[\mathrm{ps} / \mathrm{nm}]
\end{aligned}
$$

Figure 4 (a), (b) show calculated reflection dispersion of the SBLS-based multiwavelength-switchable filter at optimal settings for fast and slow axis, respectively. The maximum dispersion is calculated as $15.45[\mathrm{ps} / \mathrm{nm}]$ at both fast and slow axis. In Case I and II, the dispersion curves have mirror symmetries at principal axes.

Figure 5 (a), (b) show calculated reflection dispersion 


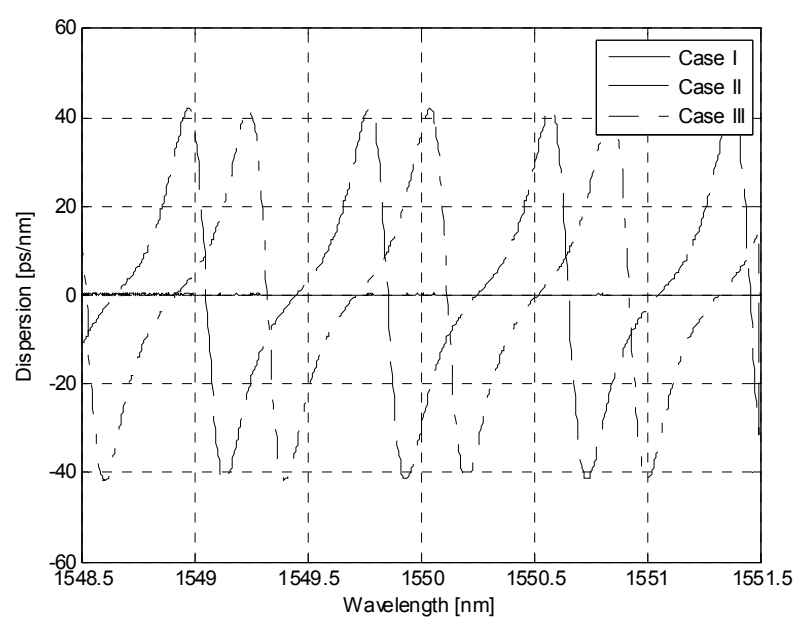

(a) fast axis

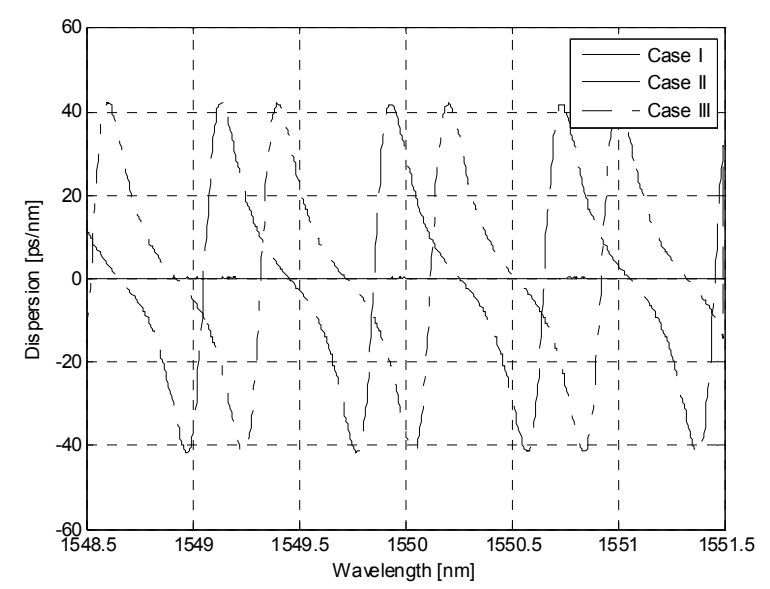

(b) slow axis

FIG. 5. Calculated reflection dispersion of the SBLS-based multiwavelength-switchable filter at non-optimal settings.

of the SBLS-based multiwavelength-switchable filter at non-optimal settings for fast and slow axis, respectively. The maximum dispersion is calculated as 41.8 [ps/nm] at both fast and slow axis. It is noticeable that in Case I (channel isolation), the dispersion is calculated to 0 over the calculation range at both fast and slow axis. In Case II and III, the dispersion curves have also mirror symmetries at principal axes.

The polarization mode dispersion (PMD) of optical devices is calculated as follows;

$$
P M D=\left|t_{g 1}-t_{g 2}\right|[\mathrm{ps}]
$$

where $t_{g 1}, t_{g 2}$ are group delays at fast and slow axis, respectively.

Figure 6 (a) shows calculated reflection PMD of the SBLS-based multiwavelength-switchable filter at optimal settings. The maximum value is 14.1 ps. Figure 6 (b) shows calculated reflection polarization mode dispersion

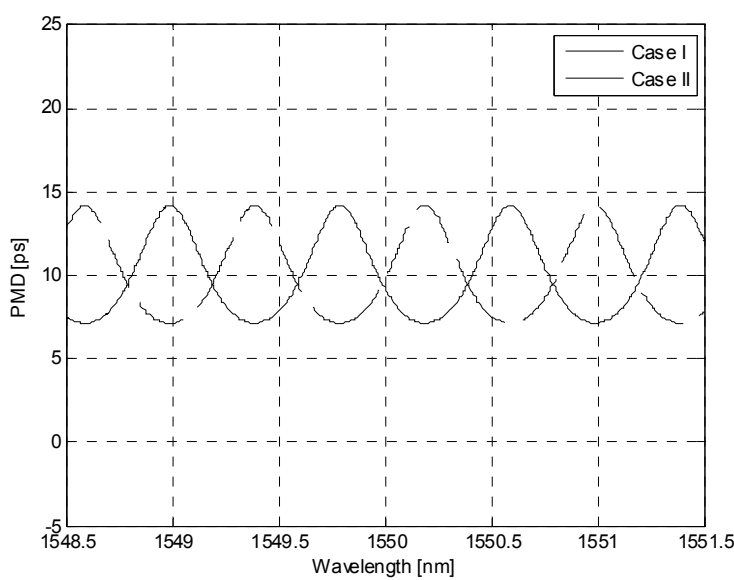

FIG. 6. (a) calculated reflection PMD of the SBLS-based multiwavelength-switchable filter at optimal settings

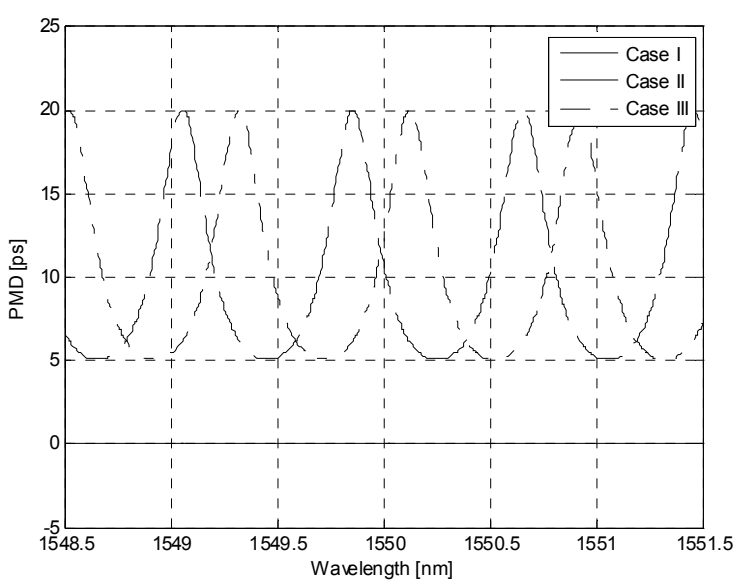

FIG. 6. (b) calculated reflection PMD of the SBLS-based multiwavelength-switchable filter at non-optimal settings

(PMD) of the SBLS-based multiwavelength-switchable filter at non-optimal settings. The maximum value is 20.0 ps. From Figure 6, we see that the PMD at optimal settings is smaller than at other ones by 5.9 ps. The dispersion is zero in the calculation range in the schemes at Case I, therefore this schemes is advantageous to dispersion-limited fiber communication systems.

\section{CONCLUSIONS}

This paper proposes a novel polarization-independent SBLS-based multiwavelength-periodic filter and presents measurements and analysis of its spectrum. The filter can be used in several schemes by adjusting the orientation angles of two quarter waveplates and the operating characteristics in reflection type are analyzed including dispersion and polarization mode dispersion at each principal axis. At optimal settings, the maximum dispersion is calculated as $15.45[\mathrm{ps} / \mathrm{nm}]$ at both fast and slow axis, and the dispersion curves have mirror symmetries at 
principal axes. At non-optimal settings, the maximum dispersion is calculated as 41.8 [ps/nm] at both fast and slow axis. It is noticeable that in channel isolation scheme, the dispersion is calculated to 0 over the calculation range at both fast and slow axis. In other schemes, the dispersion curves have also mirror symmetries at principal axes.

This filter has polarization-independent spectra but a polarization-dependent dispersion, consequently polarization mode dispersion whose values changes with operating schemes. The maximum value of PMD is 14.1 ps and 20.0 ps at optimal and non-optimal settings, respectively. The PMD-zero characteristic in the schemes at Case I is advantageous to dispersion-limited fiber communication systems.

\section{ACKNOWLEDGMENT}

The present research was conducted by the research fund of Dankook University in 2006.

\section{REFERENCES}

[1] D. B. Mortimore, "Fiber loop reflectors," J. Lightwave Technol., vol. 6, pp. 1217-1224, 1988.

[2] X. Fang and R. O. Claus, "Polarization-independent allfiber wavelength division multiplexer based on a Sagnac interferometer," Opt. Lett., vol. 20, pp. 2146-2148, 1995.

[3] X. Fang, H. Ji, C. T. Allen, K. Demarest, and L. Pelz, "A compound high-order polarization-independent birefringence filter using Sagnac interferometers," IEEE Photon. Technol. Lett., vol. 9, pp. 458-460, 1997.

[4] Y. W. Lee, J. Jung, and B. Lee, "Multiwavelength-switchable SOA-fiber ring laser based on polarization-maintaining fiber loop mirror and polarization beam splitter," IEEE Photon. Technol. Lett., vol. 16, pp. 54-56, 2004.

[5] R. C. Jones, "New calculus for the treatment of optical systems,” J. Opt. Soc. Am., vol. 31, pp. 488-492, 1941.

[6] Y. W. Lee, Study on Tunable All-Fiber Wavelength-Selective Filters and Their Applications, PhD Thesis, Seoul National University, 2004. 